\title{
Über einige deutsche Ortsnamen im französischen Volksepos.
}

Die folgenden Zeilen wollen zu den Ausführungen, die Remppis (Die Vorstellungen von Deutschland im französischen Heldenepos und Roman, Halle I9I I = Beihefte zur Zeitschr. f. rom. Phil., Heft 34, S. 12-30) der Geographie (Flüsse, Städte, Burgen) der Chansons de geste gewidmet hat, einige Nachträge bringen. ${ }^{1}$

Die hier zu besprechenden Namen gehören teils dem Rheingebiet, teils den Herzogtümern Schwaben und Sachsen an.

\section{Rheinisch-fränkische Orte.}

1. Aufalbé.

Im Doon de Manence S. 136 (V. 4497) wird ein Mitglied des Geschlechtes der Mainzer Verräter, die dem Sohne ihres angestammten Herrn, dem jungen Doon von Mainz, ${ }^{2}$ nach dem Leben trachten, bezeichnet als der Herr von Aufalbé ("le segnor d'A., im Reim auf $\dot{c}$ ). Langlois (Table des Noms propres, s. v.) weifs über diesen Namen nichts zu sagen, und auch Remppis (a.a.O. S. 27) bemerkt resigniert, dafs diese Örtlichkeit zu denen zu rechnen ist, "die von vornherein aller Erklärungsversuche spotten". Mir scheint der Versuch nicht so hoffnungslos: ich deute den Namen als Offenbach a. Main, bei Frankfurt, also gar nicht weit von Mainz, ein Ort, der schon im X. Jahrh. genannt wird. ${ }^{3}$ Aus der schon

1 Dieser Abschnitt der im allgemeinen sebr sorgfältigen Schrift ist verhältnismälsig dürftig ausgefallen, was z. T. auf der fehlerhaften Neigung des Verf.'s beruht, Eigennamen, deren Identifikation nicht von vornherein klar ist, als Phantasienamen zu betrachten. Der Umfang seiner bibliographischen Kenntnisse ist sehr bedeutend, aber manches ist ihm doch entgangen. So scheinen ihm meine Studien zu den Quellen der Chansons de geste gänzlich unbekannt geblieben zu sein. Und doch hätte er hier verschiedenes auf sein Thema bezügliches Material finden können, z. B. über Ostessin im Auberi (vgl. meinen Aufsatz $Z u$ den geschichtlichen Quellen des Auberi, Zeitschr. XXXIII, S. 34f.) oder über Rune und Colaire in den Saisnes (vgl. meine Schrift über die Sachsenkriege des französ. Volksepos S. $30 \mathrm{ff}$. und S. 50).

2 Das ist das berühmte und grofse Mainz a. Rhein, wie der Dichter V. 3183 ff. ausdrücklich hervorhebt: Maience la grant... jouste le Rim... par decoste Alemaigne (d. i. Alemannien oder Schwaben).

8 Mit diesem Offenbach a. Main konkurriert ein andrer Ort desselben Namens, gelegen in der bayrischen Pfalz, zwischen Landau und dem Rhein- 
mittelalterlichen Form Offenbach wurde Aufalbs durch Anlehnung an heidnische Ortsnamen wie namentlich Aufalerne, ein Name, der (s. Langlois, Namenbuch) im Huon de Bord. und in der Aye d'Av. oft genannt wird und dem Verf. des Doon eben aus diesen Gedichten bezw. aus einer gemeinsamen Quelle bekannt gewesen sein wird.

\section{Oupin.}

In der von Stimming (Gesellsch. für roman. Lit., Bd. 30, Dresden I912) herausgegebenen zweiten Fassung des festländischen Bueve de Hanstone, V. 4640 landen die Kaufleute, die in ihrem Schiff den Helden in seine Heimat zurückführen, ehe sie nach Köln gelangen, in Oupin (Var. Hupin) ${ }_{n}$ a set liues de Couloigne". Dieser Ort, der sonst nirgends genannt wird, dürfte zu identifizieren sein mit Oppenheim (auch Oppinheim und Uppenheim, s. Förstemann, Altdeutsch. Namenbuch II ${ }^{2}, \mathrm{Sp} .1493$ ), einer ca. $25 \mathrm{~km}$. südlich von Mainz gelegenen, im Mittelalter sehr bedeutenden und oft (seit dem 8. Jahrh.) genannten Stadt. Dafs die tatsächliche Entfernung dieses Ortes von Köln weit grölser ist als die im Gedicht angegebene (wogegen diese ungefähr zu der tatsächlichen Entfernung von Mainz stimmt), braucht natürlich von dieser Vermutung durchaus nicht abzuhalten.

\section{Montresvel.}

Im Renaut de Montauban (ed. Michelant S. 367) zieht Kaiser Karl von Montauban in Südfrankreich (Tarn-et-Garonne) gegen den in Tremoigne (Dortmund) wie ein Herzog von Sachsen 1 Hof haltenden Renaut zu Felde und langt endlich in Moniresvel bei Tremoigne an, woselbst er sein Lager aufschlägt: "Tant tindrent lor jornees qu'il sont a Montresvel, Itant pres de Tremoigne qu'il le virent a l'uel's (l. c. S. 367, V. 4-5; V. 6: Montrevel). Dieser Ort, mit dem Remppis (S. 28) ebensowenig etwas anzufangen weils wie mit den übrigen hier besprochenen, dürfte mit der kaiserlichen Burg Trifels (alte Schreibung Trivels, auch Triveles), gelegen in der jetzigen bayrischen Rheinpfalz, ca. $10 \mathrm{~km}$ westlich von Landau, zu identifizieren sein. Die sehr feste, auf steiler Berghöhe gelegene Burg wird in der deutschen Kaisergeschichte öfter genannt, so im Jahre 1113, wo der vom Kaiser Heinrich V. gefangengenommene Erzbischof Adalbert von Mainz gezwungen wurde, sie dem Kaiser auszuliefern,2 sowie im Jahre I125, wo derselbe Heinrich, dem

strom, ein Ort, der schon in Urkunden des 8. Jhds erscheint (s. Förstemann, Namenbuch $\mathrm{II}^{2}$ Sp. 1500 ), der allerdings erheblich weiter von Mainz entfernt ist als jener, aber immerhin daneben hier auch in Betracht kommt.

1 An seinem Hofe Tant i a pers et contes, le conte n'en diroie, 1. c. S. 366, V. 34 .

Vgl. Meyer v. Knonau, Fahrbücher des Deutschen Reichs unter Heinrich IV. und Heinrich V., Leipzig 1907, Bd. VI, S. 275. Die sächsischen 
Tode nahe, die Krone und die übrigen königlichen Kleinodien auf jener Feste niederlegen liefs. 1 Die Form macht gar keine Schwierigkeiten: Trivels wird in der Dichtung, mit Umstellung von s, Tresvel, und Mont ist nach dem Muster zahlreicher französischer Ortsnamen vorangestellt worden; die genaue und richtige Schreibung wäre also Mont-Tresvel.2

\section{Abroine.}

Renaut de Mont. S. 454, V. $16 \mathrm{ff}$, bei der Beschreibung des Reiseweges, den der Leichnam des Helden und Heiligen von Köln nach Dortmund zurücklegt, heilst es: Par deles une ville qui ot a non Reoigne, S'arresta li saint cors, onques n'i quist essoine; Par toute la contree dessi que vers Abroine I vinrent li malade etc. Von dem ersten der beiden in dieser Stelle genannten Orte wird noch weiter unten, bei den schwäbischen Orten, zu handeln sein. Was aber den zweiten, Abroine, ${ }^{3}$ anbetrifft, so möchte ich die Aufmerksamkeit der Fachgenossen auf die Tatsache lenken, dals der jetzt so genannte Heiligenberg, gelegen Heidelberg gegenüber am untern Nekar, dort, wo der Flufs aus dem Berggebiete des Odenwaldes in die Rheinebene eintritt, in den hier in Betracht kommenden Zeiten Abrinsberg oder, mit der ursprünglichen Form, Aburwinesberg, Aburinesberg, hiefs. Man vgl. über diesen Namen Förstemann, Altdeutsches Namenbuch $\Pi^{2}$, Sp. 2-3. Danach ist allerdings nur die Form Abrinsberg belegt (eine auf dem Berg gelegene Burg, jetzt Ruine, auch in der Form Aberinesburg), aber es erscheint zweifellos, dals das erste Element dieses Ortsnamens der Genitiv des Personennamens Aburwin ist (abar oder abur, von Förstemann mit got. abrs $=$ validus, ags. afor $=$ vehemens zusammengestellt, und wini $=$ amicus), den wir voll erhalten finden in dem Orts-

Fürsten hatten sich in dem genannten Jahre, angestiftet durch Adalbert, gegen den Kaiser empört, wurden aber unterworfen; der mächtigste derselben, Wiprecht (in den Annalen Wicpertus) ron Groitsch im Pleifsnerland, wurde gefangen genommen, zum Tode verurteilt, aber zu dreijähriger Gefangenschaft (auf der dem Kaiser soeben überlieferten Burg Trifels) begnadigt. Ein seltsames Zusammentreffen, dals in der Geschichte wie in der Dichtung diese Burg im Zusammenhang mit einem Feldzuge des Kaisers gegen die Sachsen erscheint, der mit der Unterwerfung derselben endet; seltsam auch, dafs in der Geschichte wie in der Dichtung auf Seite der Sachsen zwei Männer hervorragen, deren Namen mit Adal bezw. Wic beginnen: in der Geschichte Adalbert und Wicbert, in der Dichtung Adalhart (Aalart) und Wichart (Guichart), die beiden Brüder Renaut's. Man fühlt sich fast versucht, hier einen $\mathrm{Zu}$ sammenhang 2 wischen Geschichte und Dichtung anzunehmen.

1 S. Meyer v. Knonau, a. a. O. VII, S. 323.

2 Montrevel im Girart de Ross. und im Gaydon (s. Langlois, Namenbuch) dürfte derselbe Ort sein.

3 Das zu diesem Namen von C. Th. Müller (Zur Geographie der älteren Chansons de geste, Göttinger Dissert. 1885, S. 18) zweifelnd und ohne irgendwelche Erläuterung erwähnte oberitalienische Abrone (mir unbekannt) mufs natürlich hier aufser Betracht bleiben. 
namen Aburwinesheim (auch ohne w Aburinesheim, beide Formen belegt im VIII. Jahrh., später Ebernesheim, jetzt Ebersheim, gelegen im Süden von Mainz, Wormser Gau, s. Förstemann a. a. O.). Aus Abur(w)inesberg wurde Abroine, indem -berg dem frz. mont gleichgesetzt wurde, das ja bei Ortsnamen des frz. Volksepos öfters nach Belieben zugesetzt oder auch fortgelassen wird (vgl. oben MontTreszel $=$ Trivels) .

Der Aburulinesberg gehörte der hochberühmten Abtei Lorsch, die ca. $35 \mathrm{~km}$ nördlich desselben gelegen ist. Einer ihrer verdientesten Äbte war im XI. Jahrh. der wegen seiner christlichen Tugenden weit und breit gerühmte Reginbald, der im Jahre 1032 von Kaiser Konrad II. zum Bischof von Speyer erhoben wurde. Als Abt von Lorsch hatte er auf dem Abrinsberge ein Kloster errichtet.1 Als Bischof von Speyer entfaltete er eine grofsartige Bautätigkeit, indem er hier den nach dem Kölner berühmtesten der deutschen mittelalterlichen Dome erbaute, ${ }^{2}$ s. Bresslau in dem unten zitierten Werke II, 388.

\section{Schwabische Orte.}

\section{Reoigne.}

In der oben S. 460 zitierten Stelle des Renaut de Mont. wird eine Stadt namens Reoigne genannt, die nach den geographischen Vorstellungen des Dichters zwischen Köln und Dortmund gelegen sein muls. Dieselben sind aber sehr ungenau, denn dieser Ort ist höchst wahrscheinlich nichts anderes als das schwäbische Rheinau, in den Urkunden genannt Renaugia (auch Rinangia oder Rinowa), eine im VIII. Jahrh. von Wolfhard, Grafen von Kiburg, ${ }^{3}$ gestiftete und später föfter genannte Abtei, gelegen auf einer Rheininsel unterhalb der Stadt Schafthausen. Vgl. über diese Abtei, ihre Geschichte und ihren Namen Förstemann, Altdeutsches Namenbuch II 2 , Sp. 1249; das Geographische Lexikon von Schwaben, Ulm I801; sowie

1 Bresslau: $\mathscr{F}$ ahtö̈ücher des Deutschen Reichs unter Konrad II., Leipzig 1884, Bd. II S. 4 und Anmerk. 6 (aus Chron. Lauresh.): Monasterium in Abrinsberg a fundamentis inceptum aedificavit.

2 Wäre es zu kühn, auf die oben angeführten Umstände die Vermutung zu gründen, dals zwischen Geschichte und Dichtung hier Beziehungen stattfinden? Man vergegenwärtige sich: einerseits ein durch beiligen Wandel ausgezeichneter Kirchenfürst Reginbald, der auf dem Aburinsberg ein Kloster gebaut und sich als Erbauer eines der herrlichsten Dome einen Namen gemacht hat; andrerseits der hl. Reinhold-Renaut (der Name ist fast völlig identisch mit Reginbald), der am Bau des berühmtesten aller deutschen Dome (zu Köln) arbeitet und zu dessen Leichnam die Kranken von weither, bis von Aburinsberg (Abroine) her, kommen.

- Dies ist der älteste Vertreter dieses schwäbischen (thurgauischen) Grafengeschlechtes, dem auch jener Wernker (Wexilo) angehört, der, wie bekannt (vgl. meinen oben zitierten Aufsatz zu den Quellen des Auberi, S. 34), in der Gestalt des treuen Begleiters des Burgunders Auberi, Namens Gascelin, eine dichterische Verherrlichung gefunden hat. 
Bresslau, Jahrbücher des deutschen Reiches unter Konrad 1I., Leipzig 1879, Bd. I, S. 199. Der Name Renaugia, aus dem die franz. Namensform Reoigne einfach durch Umstellung von $n$ gebildet worden ist, wird, ebenso wie der oben behandelte Name Aburinsberg (Abroine), in einer vom Dichter benutzten geistlichen Quelle gestanden haben. Dafs beide Orte tatsächlich keineswegs auf dem Reisewege von Köln nach Dortmund liegen, kümmerte ihn durchaus nicht; wichtig war es dagegen für ihn, dafs er für die hier vorliegende Tirade, worin die Namen Couloigne und Tremoigne als Reimwörter vorkommen, zwei weitere Namen mit der durch Umstellung gebildeten Endung -oigne (-oine) gewann.

\section{Aufaï.}

Auf seiner Reise von Flandern nach Baiern kommt der Burgunder Auberi nach einer Burg mit Namen Aufä̈ ("Aus der chanson de gesle von Auberi dem Burgunder", ed. Tobler, Leipzig 1870, S. 130, 143; auch als Nom. mit flexivischem s: Li chastiax est apeles Aufaïs S. 134). Dieser Name dürfte nichts anderes sein als der aus mehreren anderen Chansons de geste, wie dem Floovant und dem Gir. de Ross. (s. Langlois, Table des noms propres), wohlbekannte Name unseres Elsals: Ausai (Aussai).1 Dem Dichter der von Tobler veröffentlichten Auberifassung war ohne Zweifel der Name Ausai, der in seiner Quelle gestanden haben murs, unbekannt; er machte ihn zunächst des Versmalses wegen aus einem zweisilbigen zu einem dreisilbigen, und er vertauschte dann den Buchstaben $s$ mit $f$, was bei der grofsen Ähnlichkeit derselben $\left(\int\right.$ und $f$ ) ja aufserordentlich leicht geschehen konnte und überdies durch andere Namen des Volksepos, wie das mehrfach vorkommende Aufalerne, nahegelegt wurde, vgl. oben S. 459 die Umgestaltung des deutschen Namens Offenbach zu Aufalbe. Dals er dabei das Ausai seiner Quelle aus einem Länder- in einen Orts- oder Burgnamen umwandelte, ist nicht verwunderlicher als die Tatsache, dafs er Baviere als Namen einer Stadt auffalst (s. Toblers Ausgabe, Register), ${ }^{2}$

1 Floovant S. 10 findet sich auch die Form Ausais, deren -s allerdings, da sie als Akkus. erscheint, fehlerhaft ist, die aber hinsichtlich dieses $s$ zu der Form Aufaïs des Auberi zu stellen ist.

2 Sollte etwa der in Aufä̈ gebietende Markgraf Tiecelin, der (Ausg. Tobler S. 143) als Auberi's Vasall erscheint und ihm als solcher bei dem Zuge nach Baiern Gefolgschaft leistet, identisch sein mit dem im Garin le Loherain als Oheim des Helden vorkommenden Tierri des Mons d'Aussai, dessen von F. Lot versuchte Identifikation mit dem Lothringerherzog Tierri $V$. von Langlois (Namenbuch, s. v. und Anm. dazu) als ganz unbegründet verworfen wird? Man erwäge, dals Tiecelin nichts andres ist als eine Deminutiv- oder Koseform zu Tierri. In historischer Beziehung kann daran erinnert werden, dals in den hier in Betracht kommenden Zeiten das Elsafs zum Herzogtum Schwaben (Alemannien) gehörte; die Vasallenschaft eines im Elsals (Aufai $=$ Ausai) gebietenden Herren gegenüber Auberi stimmt also gut zu der Tatsache, 


\section{Noigle.}

Auf dem Wege von Aufaï nach Baiern (Baiviere) kommt der Burgunder Auberi zu einer Stadt, von der die folgende Beschreibung gegeben wird (Auberi le Bourg., ed. Tobler S. 144): Une cites anchienne trouva, Noigle ot non, faile fu grant piece a: Des $V I C$. ans que Diex se bautiza; Li mur chaïrent. el la cit craventa; Desous au port un felon passaige $a^{\prime \prime}$. Da diese Schilderung einer 600 Jahre nach Christi Taufe erbauten Stadt, deren Mauern und Häuser eingefallen sind und unterhalb deren sich eine böse (gefährliche) Talenge! befindet, auf mich keineswegs den märchenhaft-phantastischen Eindruck gemacht hat, den Remppis (l.c. S. 30) daraus entnehmen zu müssen glaubt, hat sie mich auch nicht, wie ihn, davon abgehalten, mich auf dem hier in Betracht kommenden geographischen Gebiet, d. i. dem schwäbischen (zwischen $A u f a \ddot{z}=$ Ausai, d. i. Elsa/s, undBaiern), nach einer Örtlichkeit umzusehen, auf die jene Schilderung passen würde. Ein durchaus sicheres Ergebnis ist dabei allerdings nicht herausgekommen; einen starken Anspruch auf Beachtung aber scheint mir die Stadt Rottenburg, im Südwesten von Tübingen am Neckar gelegen, $z u$ haben. Man erwäge die folgenden Umstände. Einmal: Rottenburg ist eine uralte Stadt, da auf ihrem Boden schon eine römische Kolonie stand, namens Sumlocenne (Sumelocenna), von der in der Neuzeit bedeutende Überreste ans Tageslicht gefördert worden sind. Diese römische Stadt wurde, wahrscheinlich im Jahre $369 \mathrm{n}$. Chr., durch eine Überschwemmung des Neckars zerstört (vgl. Ammianus Marcellinus ed. Gardthausen XXVIII, 2, 2-4) ${ }^{2}$; sie wurde dann, in der frühmittelalterlichen, merovingischen Zeit wieder aufgebaut, aber im Jahre I 112 durch Erdbeben und zugleich Überschwemmung abermals zerstört, endlich im XIII. Jahrh. wieder aufgebaut; sie erhielt jetzt den Namen Rottenburg, während sie bis zu der Katastrophe des Jahres II 2 Landskron (oder Landsort) geheilsen hatte. Bei Ausgrabungen hat man nicht nur Reste der römischen, sondern, durch eine dicke darüberliegende Lehmschicht davon getrennt, auch solche der frühmittelalterlichen (merowingischen) Stadtmauer blofsgelegt, und Reste der im XIII. Jahrh. erbauten sind noch jetzt allen sichtbar. An die Katastrophe des Jahres 1112 erinnert noch jetzt eine auf einem alten Mauerrest angebrachte und im Jahre 1602 erneuerte Inschrift, wonach ndie Stadt Landskron oder Landsort anno Christi 1112 den dritten des Jenners durch Erdbidem (d. i. Erdbeben)

dafs in dieser Heldengestalt des französischen Volksepos ein gut Teil von dem geschichtlichen Herzog Ernst von Schwaben steckt (vgl. hierüber meinen oben zitierten Artikel der Zeitschr., S. 29 ff.).

1 Port bedeutet hier nicht, wie Remppis will, „Hafen“ ,sondern „Engpafs".

2 Hier wird erzählt, dafs in dem genannten Jahre Kaiser Valentinian. dem Neckar ein anderes Bett gab, um Befestigungen, die er an seinem Ufer (bei Sumlocenne) angelegt hatte, vor der Zerstörung durch den ausgeuferten wilden Flufs zu bewahren. 
und Gewässer untergegangen und a. $127 \mathrm{I}$ vom Grafen Albrecht von Hohenberg wieder aufgebaut und Rottenburg genannt worden ist". Ich möchte also die Vermutung wagen, dafs wir bei der alten Stadt des ${ }_{n}$ Auberi" ${ }^{\text {', }}$ deren Mauern zerfallen sind, an einen Ort am oberen Neckar zu denken haben, der durch eine der hier so häufigen Überschwemmungen oder auch Erdbeben (Schwaben hatte vom XI.-XII. Jahrh. eine Erdoebenperiode!) zerstört worden ist, vornehmlich aber an Landsort-Rottenburg.1 Einwenden könnte man freilich, dals keine zeitgenössische Quelle (Urkunde oder Chronik) von der Zerstörung dieser Stadt berichtet, sondern erst viel spätere (die älteste, in dem Geschichtsbuch eines Bürgermeisters von Horb, namens Besenfelder [ $†$ 1470] stammt aus dem XV. Jahrh.); dagegen ist aber $\mathrm{zu}$ bemerken, dals der um die alte Geschiehte seiner Heimatstadt hochverdiente Arzt Dr. Paradeis in Rottenburg durch seine hier vorgenommener Ausgrabungen, wodurch, abgesehen von der Römerstadt, auch Reste einer durch Neckarüberschwemmung (bezeugt durch zahlreiche Muschelschalen) zu Grunde gegangenen frühmittelalterlichen Stadt ans Tageslicht gekommen sind, den Mangel gleichzeitiger geschriebener Quellen in einer Weise ersetzt hat, dafs an der Tatsächlichkeit des hier zur Stütze herangezogenen Ereignisses (Zerstörung des frühmittelalterlichen Landskron-Rottenburg) kaum zu zweifeln ist."

Was nun aber den in dem französischen Gedicht stehenden Namen Noigle betrifft, so vermute ich, dals derselbe eigentlich nichts anderes ist als der Name des Flusses, der hier so oft genannt werden mulste: des Neckars, sei es dafs der bei den Alten (vgl. Stälin, Wirtemberg. Gesch. I, 133) gebrauchte Name Nicer (Acc. Nicrum) oder Niger (Acc. Nigrum), sei es dals der mittelalterlich-deutsche Name Nekar (daneben auch mit 0 in der 2. Silbe: Nechor oder als Fem. Nechora, s. Förstemann, Namenbuch II'2, Sp. 1146 ) dem französischen Dichter bekannt geworden ist. $\mathrm{Dafs}$ er daraus den Namen einer Stadt gemacht hat (wobei er eigentlich Landskron-

1 Für diesen letzteren Ort spricht auch der Umstand, dafs ganz in der Nähe von Rattenburg der Neakar eine gefährliabe, d. h. leicht Úberschwemmungen hervorrufende Talenge hat (vgl. Paradeis in den Reutlinger Geschichtsblättern XVIII, S. 43), womit die Bemerkung des altfranz. Dichters: Desous au port un felon passaige a (nunten in der Talenge ist eine gefährliche Stelle") zusammenzuhalten ist.

2 Die Angaben die hier über die Geschichte Rottenburg's gemacht worden sind, verdanke ich zum grofsen Teil der Liebenswürdigkeit des Herrn Dr. Paradeis, dem ich auch an dieser Stelle meinen Dank dafür ausspreche; ebenso habe ich zu danken der Leitung der Tübinger Universitätsbibliothek, die mich mit einschlägiger Literatur versehen hat, besonders Herrn Dr. Ha u ber. Von Schriften über die Geschichte Rottenburg's ist zu nennen v. Jaumann: Colonia Sumlocenne oder Rottenburg unter den Römern, Stuttg. u. Tübingen 1840; Hassler: Chronik von Rottenburg und Ehingen, Rottenburg 1819; namentlich aber Paradeis, der in den Reutlinger Geschichtsblättern Jahrgang XVIII (I907) S. 23 ff und in den Fundberichten aus Schwaben (1912) S. 47 ff. die Ergebnisse seiner höchst erfolgreichen archäologischen Forschungen mitgeteilt hat. 
Rottenburg im Sinne hatte) ist einem Dichter wohl zuzutrauen, der (s. oben S. 462) Baiern zu einer Stadt und das Elsafs zu einer Burg macht.1

\section{Jenor.}

Im Floovant (S. 31) wird erzählt, dafs der aus Ausai (d. i. Elsafs) kommende Richier, der Getreue des aus Frankreich verbannten Helden, den aus seinem Lande Baviere von den Heiden (das sind eigentlich wohl die Ungarn des X. Jahrh., wie im Auberi) vertriebenen Herzog Emelon am Ufer des Flusses Jenor findet. Auch dieser Name könnte, so vermute ich, eine Umgestaltung des Flufsnamens Neckar sein, ebenso wie Noigle in Auberi, nur in anderer Weise. Nechor (über diese Namensform s. oben bei Noigle, S. 464) könnte zu *Chenor umgestellt, dies aber zu Jenor verändert worden sein.

\section{Săchsisch-thuringische Orte.}

\section{Retefor.}

Diese Stadt wird in der von Stimming herausgegebenen anglonorm. Fassung des Bueve de Hanstone V. 75 und 77 als Aufenthaltsort des deutschen Kaisers Doon genannt. Der Herausgeber (Anm. zu V. 75) erklärt, nicht zu wissen, welche Stadt damit gemeint ist. Es könnte m. E. Erfurt oder (der ursprüngliche Name, s. Förstemann, Namenbuch II 2, Sp. I19) Erpesfurt sein, vom X. bis XUI. Jahrh. eine der bedeutendsten Städte Deutschlands und sehr häufig Aufenthaltsort der deutschen Kaiser. In der nordischen Fassung fehlt der Name, in der welschen steht ein ganz anderer (Calys, was einigermalsen an Goslar erinnert, ein Ort, der in der Chans. des Saisnes als Colaire vorkommt, vgl. meine Schrift über die Sachsenkriege S. 50), in der englischen (eine Fassung, die nach dem von Stimming S. CLXXVI der Einl. aufgestellten Stammbaum besondere Autorität beanspruchen kann) stehen die Formen Rifoun, Rapain, Repain. Danach möchte ich vermuten, dafs in der Fassung, die der anglonormannischen sowie den damit zusammenhängenden fremdländischen Bearbeitungen zu Grunde gelegen haben muls, eine Form *Repefort gestanden hat, welche der deutschen Namensform (Erpesfurt) ganz nahe steht.

1 Das $l$ der Form Noigle exinnert auffallend an den ähnlichen Namen einer in der Nähe von Rottenburg gelegenen Stadt: Nagalta, jetzt Nagold, gelegen an dem gleichnamigen Flurs in einem tief eingeschnittenen Tale des Schwarzwaldes (sehr alt, bezeugt seit dem VIII. Jahrh., vgl. Förstemann, Namenbuch II2 Sp. II39; Stälin, Wirtemberg. Gesch. I, 302). Sollte etwa auch dieser Name mitbeteiligt sein, also Noigle $=$ Nigrum (bzw. dtsch. Nekor) vermengt mit Nagalta? 


\section{Valentin, Val Saint Dié und Ballete (Balide).}

Renaut de Mont. S. 264 fordert Kaiser Karl seinen Neffen Roland auf, den gefangenen Richart, Renauts Bruder, zu hängen, und verspricht $\mathrm{ihm}$ dafür verschiedene Länder zu Lehen zu geben: Et vos, biaus nies Rollans, l'empereres a dit, Or le vos covient pandre, puisque vos l'aves pris; Et je vos en donrai Couloigne sor le Rin, Et Ballet et Tremoigne et Hollande a tenir, Et le Val Saint Dié, i'est $\cdot I$ lius barbarins, Et trestoute la terre desi a Valentin. Von den hier genannten Orten bezw. Ländern sind bisher Ballet, Saint Die und Valentin unerklärt geblieben. Sie dürften, ebenso wie Couloigne, Tremoigne und Hollande, im nördlichen Deutschland zu suchen sein. Bei Valentin könnte man an Avalterre (auch Avalois) denken, wie bekanntlich im frz. Volksepos die Niederlande bezeichnet werden. Auf ein anlautendes $A$ mehr oder weniger und auf die genaue Endung kommt es den altfranzösischen Volksdichtern oder ihren Abschreibern bei fremden Ortsnamen sehr wenig an; wird doch sogar auch für den französischen Ortsnamen Saint-Vallerin (Saint-Valery-sur-Somme) von einem Abschreiber ohne viel Federlesen die Namensform Valentin eingesetzt (im Girbert de Mes, Rom. Stud. I, 476; vgl. Langlois, Noms Propres, s. v. Saint Valery).

Was dann den Namen Val Saint Dié anbetrift, so haben die Gelehrten, die sich bisher mit diesem Namen beschäftigt haben, ohne weiteres angenommen, dals er mit dem bekannten franz. Städtenamen Saint-Die (Dép. Vosges) identisch sei. So Langlois, Noms propres, s. v.) und auch Remppis, der a.a. O. S. 28 zu der oben zitierten Stelle des Renaut bemerkt: "Schon die Zusammenstellung der niederrheinischen Gebiete mit dem in den Vogesen gelegenen Val Saint Dié zeigt, dals wir diese Angaben nicht allzu ernst nehmen dürfen ". Gegen diese bei oberflächlicher Betrachtung ja allerdings sehr naheliegende Auffassung des Namens hat aber der Dichter selbst Verwahrung eingelegt durch den Zusatz: ${ }_{n}$ 'est uns lius barbarins", welcher deutlich und bestimmt darauf hinweist, dals der Qrt, um den es sich hier handelt, mit dem in den Vogesen gelegenen durchaus nicht identisch ist; denn "lius barbarins" kann hier sicher nur bedeuten "im Heidenlande gelegener Ort 1; wir werden also diesen Ort im heidnischen Deutschland und demgemäls im Sachsenlande zu suchen haben. Und da möchte ich die Vermutung wagen, dafs in der von dem Dichter des Renaut hier benutzten (mündlichen oder schriftlichen) Quelle von dem

1 Der Ausdruck lius barbarins erinnert an den von Ammian, beim Feldzug Valentinian's gegen die Alemannen, gebrauchten: trans Rhenum in monte Piri, qui barbaricus locus est (XXVIII, 2, 5); die Bedeutung ist natürlich nicht ganz dieselbe: nicht ${ }_{n}^{\text {im Heidenlande }}$, sondern ${ }_{n}$ im Barbarenlande (Germanien jenseit des Kheins) gelegen". Wie der im Dép. der Nièvre gelegene französische Ortsname Barbarin (erwähnt von Godefroy, s. v.) zu deuten ist, geht uns hier nichts an. 
Lande des Markgrafen Dedi die Rede war, eines wackeren Fürsten aus dem wettinischen Geschlecht, der unter der Regierung des Kaisers Konrad III, nämlich seit dem Jahre 1034 in der sächischen Ostmark (zwischen der unteren Saale und der mittleren Spree) waltete und im Jahre 1046, nach dem Tode seines Oheims, des Markgrafen Eckard II. von Meifsen,1 auch den gröfsten Teil der Besitzungen desselben erbte, vgl. Giesebrecht, Gesch. d. dtschen. Kaiserzeit II 4, S. 306; Bresslau, Jahrb. des deutschen Reiches unter Konr. II., 2. Bd., S. 129. Dafs der Dichter aus dem Lande eines Dedi ein Val Saint Dié machte, d. h. einen fremden sächsischen Namen einem aus der französischen Geographie bekannten gleichstellte, ist eine Umdeutung, die, der Volksetymologie vergleichbar, durchaus im Geist der naiven Volksepik liegt.

Was endlich den Namen Ballet betrifft, für den meiner Vermutung nach Ballte oder Balete zu lesen sein wird, so ist dies m. E. nichts anderes als Paliti (auch Palide; später auch mit 0 und e: Poledi, Polethe, jetzt Pöhlde, s. Förstemann, Namenbuch $\mathrm{II}^{2}$, Sp. I187), kaiserliche Burg (Pfalz) und Kloster, gelegen am Südabhange des Harzes, ca. $30 \mathrm{~km}$ nordwestlich von Nordhausen. Der Ort war den sächsischen Kaisern überaus lieb und wert; sie weilten hier mit Vorliebe; aber auch noch unter dem fränkischen Kaiser Konrad II. wird der Ort öfters, auch als Zusammenkunftsort des Kaisers und der Reichstürsten, erwähnt, s. Giesebrecht a.a. O. II 4 Register, Bresslau, Jahrb. des deutschen Reiches unter Konr. II., Register.

Dals aus Paliti die Namensform Ballet[e], mit $B$, wurde, hat nichts zu sagen, kann übrigens auch als Anlehnung an den Namen des dem Volksepos bekannten Sachsenkönigs Balan (oder des gleich noch zu erwähnenden Baligant) gedeutet werden.

Mit diesem Ballet [e] wird nun auch identisch sein der bisher unerklärt gebliebene Name Balide, im Oxforder Roland V. 3230, wo berichtet wird, dals des Admirals Baligant zehnter Heerhaufe von Kriegern aus Balide gebildet wird. Wenn Stengel im Namenverzeichnis seiner Rolandausgabe zu dem Namen die Erklärung gibt: "Stadt im Orient", so ist das lediglich eine Annahme und zwar eine, bei der orientalischen Herkunft des Admirals, naheliegende, aber deswegen noch keineswegs richtige: die als Bestandteil desselben Heeres V. 3226 genannten Sorbre sind ja bekanntlich auch

1 Dieser Eckard, ein hochverdienter und dem Kaiser Konrad II. sehr nahestehender Fürst, einer der mächtigsten in Sachsen, scheint in dem im Auberi (ed. Tobler S. 143) erwähnten Egart, König von Sachsen, eine Spur hinterlassen zu haben, zu dem sich zu begeben Gascelin seinem Oheim Auberi rät. Freilich kommt daneben auch der (irre ich nicht, schon von anderer Seite herangezogene), grolse" Markgraf Eckard I. von Meilsen (+1002) in Betracht; zu Gunsten Eckard's II. spricht aber der Umstand, dafs er, ebenso wie Kaiser Konrad II., ein Zeitgenosse des Herzogs Ernst II. von Schwaben war, der, wie bekannt (vgl. meinen oben zitierten Aufsatz), der franz. Dichtung eben die Gestalt des Burgunders Auberi geliehen hat. 
keine Orientalen, sondern richtige Europäer, nämlich die Sorben, deren Gebiet, östlich der Saale und Unstrut, gar nicht sehr weit von diesem sächsischen Balide = Palithi gelegen war.

Nun ist es aber eine bekannte Tatsache, dafs die Sachsen, auch nachdem sie längst christlich geworden waren, vom französ. Volksepos immer noch als Heiden betrachtet werden. Es wird also in keiner Weise befremden können, dafs, wie die noch im $\mathrm{XII}$. Jahrh. heidnischen Sorben, so auch die Bewohner des erst seit dem X. Jahrh. durch seine christlich-sächsischen Herrscher bekannt gewordenen Balide $=$ Paliti im Rolandsliede als Heiden und Anhänger des Admirals Baligant aufgefalst wurden.

F. Settrgast. 